\title{
New Valid Inequalities for the Fixed-Charge and Single-Node Flow Polytopes
}

\author{
Adam N. Letchford* Georgia Souli ${ }^{\dagger}$ \\ To appear in Operations Research Letters
}

\begin{abstract}
The most effective software packages for solving mixed 0-1 linear programs use strong valid linear inequalities derived from polyhedral theory. We introduce a new procedure which enables one to take known valid inequalities for the knapsack polytope, and convert them into valid inequalities for the fixed-charge and single-node flow polytopes. The resulting inequalities are very different from the previously known inequalities (such as flow cover and flow pack inequalities), and define facets under certain conditions.
\end{abstract}

Keywords: polyhedral combinatorics; branch-and-cut; mixed-integer linear programming

\section{Introduction}

Polyhedral methods have proven to be remarkably useful for solving pure and mixed 0-1 linear programs (see, e.g., [5, 6]). In the case of large, sparse instances without special structure, three families of polytopes have proven to be of particular importance: the knapsack, fixed-charge and single-node flow polytopes. The knapsack polytope is the convex hull of vectors $y \in$ $\{0,1\}^{n}$ satisfying

$$
\sum_{i \in N} a_{j} y_{j} \leq b
$$

where $b$ and the $a_{j}$ are positive integers, and $N$ denotes $\{1, \ldots, n\}$ [3, 16]. The fixed-charge polytope is the convex hull of pairs $(x, y) \in \mathbb{R}_{+}^{n} \times\{0,1\}^{n}$ satisfying

$$
\begin{aligned}
& \sum_{j \in N} x_{j} \leq d \\
& x_{j} \leq u_{j} y_{j}
\end{aligned} \quad(j \in N),
$$

\footnotetext{
${ }^{*}$ Corresponding author. Department of Management Science, Lancaster University, Lancaster LA1 4YX, United Kingdom. E-mail: a.n.letchford@lancaster.ac.uk

${ }^{\dagger}$ STOR-i Centre for Doctoral Training, Lancaster University, Lancaster LA1 4YR, United Kingdom. E-mail: G.Souli@lancaster.ac.uk
} 
where $d$ and the $u_{j}$ are positive integers [11. Finally, the single-node flow polytope is the convex hull of pairs $(x, y) \in \mathbb{R}_{+}^{n} \times\{0,1\}^{n}$ satisfying

$$
\begin{aligned}
& \sum_{j \in N^{+}} x_{j}-\sum_{j \in N^{-}} x_{j} \leq d \\
& \ell_{j} y_{j} \leq x_{j} \leq u_{j} y_{j}
\end{aligned} \quad\left(j \in N^{+} \cup N^{-}\right),
$$

where $d$ and the $u_{j}$ are again positive integers, the $\ell_{j}$ are non-negative integers, $N^{+}$and $N^{-}$are disjoint sets, and $n$ now denotes $\left|N^{+} \cup N^{-}\right|[13$.

Several families of valid linear inequalities are known for the knapsack polytope, including cover, extended cover and lifted cover inequalities [3, 16, weight inequalities [15] and lifted pack inequalities [2, 10]. Inequalities for the fixed-charge polytope include flow cover inequalities [11] and lifted flow cover inequalities [8, 9]. Inequalities for the single-node flow polytope include generalized flow cover inequalities [13, 14, reverse flow cover inequalities [12, lifted generalised flow cover inequalities [8] and lifted flow pack inequalities [1]. Inequalities like these have proven to be so useful that many of them are now generated by default in the leading integer programming solvers (such as CPLEX, Gurobi and Xpress).

The purpose of this note is to present a procedure which enables one to take valid inequalities for the knapsack polytope and convert them into valid inequalities for the fixed-charge and single-node flow polytopes. We call the resulting inequalities rotated knapsack inequalities or RKIs. Even if one applies our procedure to simple inequalities for the knapsack polytope, such as cover inequalities, one can still obtain new and non-trivial inequalities for the other polytopes.

The paper is structured as follows. In Section 2, we review some of the well-known inequalities for our three families of polytopes. In Section 3 , we present our procedure for the fixed-charge polytope, show that the resulting inequalities can define facets, and examine the special cases that arise when the initial inequality is a cover or extended cover inequality. In Section 4 , we extend our procedure to the single-node flow polytope. Finally, Section 5 includes some concluding remarks and suggestions for further research.

\section{Literature Review}

We now review the literature. The following subsections are concerned with valid inequalities for the knapsack polytope, the fixed-charge polytope and the the single-node flow polytope.

\subsection{Knapsack polytope}

As mentioned above, many families of inequalities are known for the knapsack polytope. For brevity, we recall here only a few results from [3, 16]. A set $C \subseteq N$ is called a cover if $\sum_{j \in C} a_{j}>b$. If $C$ is a cover, then the cover 
inequality $\sum_{j \in C} y_{j} \leq|C|-1$ is valid. The strongest cover inequalities are obtained when $C$ is minimal (i.e., no proper subset of $C$ is a cover).

Cover inequalities can be strengthened in various ways. For example, let $a^{*}=\max _{j \in C} a_{j}$ and let $E=\left\{j \in N \backslash C: a_{j} \geq a^{*}\right\}$ be the extension of $C$. The extended cover inequality takes the form

$$
\sum_{j \in C \cup E} y_{j} \leq|C|-1
$$

\section{$2.2 \quad$ Fixed-charge polytope}

Padberg et al. [11] presented two families of inequalities for the fixed-charge polytope. The inequalities of the first family are derived as follows. A set $F \subseteq N$ is called a flow cover if $\sum_{j \in F} u_{j}>d$. Given a flow cover $F$ and a (possibly empty) set $L \subseteq N \backslash F$, we let $\lambda$ denote $\sum_{j \in F} u_{j}-d$ and $u^{+}$denote $\max _{j \in F} u_{j}$. The following flow cover inequality is valid:

$$
\sum_{j \in F \cup L} x_{j} \leq d-\sum_{j \in F} \alpha_{j}\left(1-y_{j}\right)+\sum_{j \in L} \alpha_{j} y_{j}
$$

where $\alpha_{j}$ is $\max \left\{0, u_{j}-\lambda\right\}$ for $j \in F$, and $\max \left\{u^{+}, u_{j}\right\}-\lambda$ for $j \in L$. The flow cover inequalities were slightly strengthened in [8], yielding lifted flow cover inequalities.

The second family is very different. Let $P^{=}$be the face of the fixedcharge polytope obtained by setting the inequality (1) to equality. One can check that, if $(x, y) \in P^{=}$, then $y$ must lie within the knapsack polytope

$$
K=\operatorname{conv}\left\{y \in\{0,1\}^{n}: \sum_{j \in N} u_{j} y_{j} \geq d\right\} .
$$

Let $\alpha^{T} y \geq \beta$ be any valid inequality for $K$ with $\alpha \in \mathbb{Z}_{+}^{n}$ and $\beta \in \mathbb{Z}_{+}$. It is shown in [11] that there exists a positive rational $\delta$ such that the inequality

$$
\sum_{j \in N} x_{j} \leq d+\delta\left(\sum_{j \in N} \alpha_{j} y_{j}-\beta\right)
$$

is valid for the fixed-charge polytope. To the best of our knowledge, this procedure has received no attention in the literature.

\subsection{Single-node flow polytope}

Van Roy \& Wolsey [13] extended the flow cover inequalities to the singlenode flow polytope. Now, a pair $\left(F^{+}, F^{-}\right)$is called a generalised flow cover if $F^{+} \subseteq N^{+}, F^{-} \subseteq N^{-}$and $\sum_{j \in F^{+}} u_{j}-\sum_{j \in F^{-}} \ell_{j}>d$. Given a generalised 
flow cover $\left(F^{+}, F^{-}\right)$and sets $L^{+} \subseteq N^{+} \backslash F^{+}$and $L^{-} \subseteq N^{-} \backslash F^{-}$, one can construct a valid inequality of the form

$$
\begin{gathered}
\sum_{j \in F^{+} \cup L^{+}} x_{j}-\sum_{j \in N^{-}} x_{j} \leq d-\sum_{j \in F^{+}} \alpha_{j}\left(1-y_{j}\right)+\sum_{j \in L^{+}} \alpha_{j} y_{j} \\
+\sum_{j \in F^{-}} \alpha_{j}\left(1-y_{j}\right)-\sum_{j \in L^{-}} \alpha_{j} y_{j},
\end{gathered}
$$

where $\alpha_{j} \in \mathbb{Z}_{+}$for $j \in F^{+} \cup L^{+} \cup F^{-} \cup L^{-}$. (For brevity, we skip the details on how to compute the $\alpha_{j}$.) These are called generalised flow cover (GFC) inequalities. They have been generalised and strengthened in various ways $[8,9,12,13]$. A related family of inequalities, the lifted flow pack inequalities, were studied by Atamtürk [1].

\section{$3 \quad$ Fixed-Charge Polytope}

In this section, we consider the fixed-charge polytope. Subsection 3.1 presents our procedure for generating inequalities, Subsection 3.2 gives a sufficient condition for the resulting inequalities to define facets, and Subsection 3.3 analyses two special cases.

\subsection{General procedure}

Let $P$ denote the fixed-charge polytope and $F \subseteq N$ be a flow cover. The inequality $\sum_{j \in F} x_{j} \leq d$ is trivially valid for $P$. Let $P^{=}$be the face of $P$ obtained by setting this inequality to equality. That is,

$$
P^{=}:=\operatorname{conv}\left\{(x, y) \in \mathbb{R}_{+}^{n} \times\{0,1\}^{n}:\left\{1,,\left\{2,, \sum_{j \in F} x_{j}=d\right\} .\right.\right.
$$

One can check that the following inequality is valid for $P^{=}$:

$$
\sum_{j \in F} u_{j} y_{j} \geq d
$$

Let $\bar{y}_{j}=1-y_{j}$ for all $j \in F$. Then, we can write (5) as

$$
\sum_{j \in F} u_{j} \bar{y}_{j} \leq \sum_{j \in F} u_{j}-d
$$

and define the restricted knapsack polytope

$$
K=\operatorname{conv}\left\{\bar{y} \in\{0,1\}^{F}: \sum_{j \in F} u_{j} \bar{y}_{j} \leq \sum_{j \in F} u_{j}-d\right\} .
$$




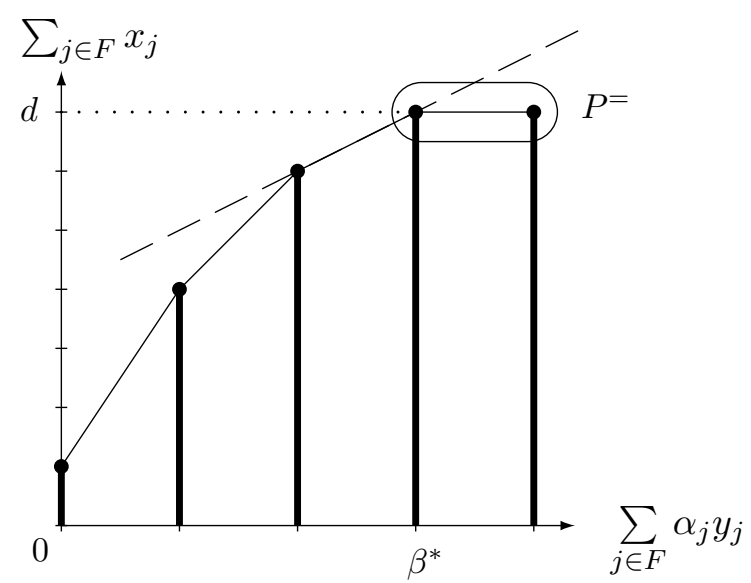

Figure 1: Projection of feasible points onto 2-dimensional subspace.

Let $\alpha^{T} \bar{y} \leq \beta$ be a supporting valid inequality for $K$ with $\alpha \in \mathbb{Z}_{+}^{F}$ and $\beta$ a positive integer. (All non-trivial valid inequalities for $K$ have this form; see [16].) Complementing the $\bar{y}_{j}$ variables, and writing $\beta^{*}=\sum_{j \in F} \alpha_{j}-\beta$, we obtain the inequality

$$
\sum_{j \in F} \alpha_{j} y_{j} \geq \beta^{*}
$$

which is valid and supporting for $P^{=}$. Our goal is to 'rotate' this inequality, in order to make it valid and supporting for $P$.

At this point, it is helpful to project $P$ and $P^{=}$onto a 2-dimensional subspace, having $\sum_{j \in F} x_{j}$ and the left-hand side of (7) as axes. This is illustrated in Figure 1. The thick vertical lines represent feasible solutions in $P$, and the horizontal line inside the ellipse at the top-right represents $P^{=}$. One can see that the inequality $(7)$ is valid for $P^{=}$but not for $P$. Moreover, given that the inequality (7) is supporting for $P^{=}$, any feasible solution satisfying $\sum_{j \in F} \alpha_{j} y_{j}<\beta^{*}$ also satisfies $\sum_{j \in F} x_{j}<d$. Therefore, there exists a positive rational number $\delta$ such that the inequality

$$
\sum_{j \in F} x_{j} \leq d+\delta\left(\sum_{j \in F} \alpha_{j} y_{j}-\beta^{*}\right)
$$

is valid and supporting for $P$. Setting $\delta$ to the largest such value yields the desired rotated knapsack inequality (RKI), which is represented by a dashed line in the figure.

In order to determine $\delta$, we define the function $\phi: \mathbb{Z}_{+} \rightarrow\{0,1, \ldots, d\}$ with

$$
\phi(t)=\max \left\{\sum_{j \in F} x_{j}: \square,(2), \sum_{j \in F} \alpha_{j} y_{j} \leq t,(x, y) \in \mathbb{R}_{+}^{F} \times\{0,1\}^{F}\right\} .
$$


By definition, $\phi$ is non-decreasing in $t$. Also, from the definition of $\beta^{*}$, and the fact that the inequality (7) is supporting for $P^{=}$, we have $\phi(t)=d$ if and only if $t \geq \beta^{*}$. Moreover, for $t=0, \ldots, \beta^{*}-1$, the constraint (1) is redundant, and we have:

$$
\phi(t)=\max \left\{\sum_{j \in F} u_{j} y_{j}: \sum_{j \in F} \alpha_{j} y_{j} \leq t, y \in\{0,1\}^{F}\right\} .
$$

The problem of determining $\phi(t)$ for $t=0, \ldots, \beta^{*}-1$ is therefore a parametric $0-1$ knapsack problem. It can be solved in $O\left(|F| \min \left\{d, \beta^{*}\right\}\right)$ time by dynamic programming. Once this has been done, one can compute $\delta$ easily, in $O\left(\beta^{*}\right)$ time, as follows:

$$
\delta=\min _{0 \leq t<\beta^{*}}\left\{\frac{d-\phi(t)}{\beta^{*}-t}\right\}
$$

\section{$3.2 \quad$ Facet-defining RKIs}

A natural question is to determine conditions under which RKIs define facets of $P$. The following proposition gives a sufficient condition. (We remark that a similar condition was given in [4] for rank inequalities to define facets of stable set polytopes.)

Proposition 1 Suppose the inequality $\sum_{j \in F} \alpha_{j} \bar{y}_{j} \leq \beta$, with $\alpha \in \mathbb{Z}_{+}^{F}$ and $\beta$ a positive integer, defines a facet of $K$. Let $G$ be a graph defined as follows. The vertex set is $F$. For all pairs $\{i, j\} \subset F$, the edge $\{i, j\}$ is present in $G$ if and only if there exists a vector $\bar{y} \in\{0,1\}^{F}$ satisfying

$$
\bar{y}_{i}=\bar{y}_{j}=0, \sum_{j \in F} \alpha_{j} \bar{y}_{j}=\beta, \text { and } \sum_{j \in F} u_{j} \bar{y}_{j}<\sum_{j \in F} u_{j}-d .
$$

If $G$ is connected, then the resulting $R K I$ defines a facet of $P$.

Proof. For brevity, we only sketch the proof. Let us call a pair $(x, y) \in$ $\mathbb{R}_{+}^{n} \times\{0,1\}^{n}$ a 'root' if it satisfies the RKI at equality. Note that the roots are of two types: (i) those with $\sum_{j \in F} \alpha_{j} y_{j}=\beta^{*}, \sum_{j \in F} x_{j}=d$, and $x_{j}=0$ for $j \in N \backslash F$, and (ii) those with $\sum_{j \in F} \alpha_{j} y_{j}<\beta^{*}$ and $\sum_{j \in F} x_{j}<d$. Now, since the original inequality defines a facet of $K$, there exist $|F|$ affinely independent roots of the first type. Without loss of generality, we assume that these roots have $y_{j}=0$ for $j \in N \backslash F$. So we can construct $n-|F|$ additional affinely independent roots of the first type, by changing $y_{j}$ from 0 to 1 for each $j \in N \backslash F$ in turn. We now have $n$ affinely independent roots of the first type.

Now, let $T$ be a spanning tree in $G$, and note that it has $|F|-1$ edges. From the definition of $G$, it follows that, for each edge $\{i, j\} \in T$, there exists a root of the first type, such that $y_{i}=y_{j}=1$ and $\sum_{j \in F} u_{j} y_{j}>d$. 
Without loss of generality, we can assume that this root satisfies $x_{i} \in\left(0, u_{i}\right)$ and $x_{j} \in\left(0, u_{j}\right)$. If this root is not affinely independent of the roots that we have seen so far, then we can make it affinely independent by increasing $x_{i}$ by some small quantity $\epsilon$, and decreasing $x_{j}$ by $\epsilon$. In this way, we construct $|F|-1$ additional affinely independent roots of the first type.

Next, observe that there exists a root of the second type for which $x_{j}=0$ and $y_{j}=1$ for all $j \in N \backslash F$, and this root is affinely independent of the previous ones. Moreover, for any $j \in N \backslash F$, we can construct another root of the second type by increasing $x_{j}$ by some small quantity $\epsilon$. In this way, we obtain an additional $n-|F|$ affinely independent roots.

We illustrate this theory with an example.

Example 1: Let $n=6, d=10$ and $u=(1,3,3,3,5,5)$. If we let $F=\{2,3,4,5,6\}$, we have

$$
K=\left\{\bar{y} \in\{0,1\}^{F}: 3 \bar{y}_{2}+3 \bar{y}_{3}+3 \bar{y}_{4}+5 \bar{y}_{5}+5 \bar{y}_{6} \leq 9\right\} .
$$

The inequality

$$
\bar{y}_{2}+\bar{y}_{3}+\bar{y}_{4}+2 \bar{y}_{5}+2 \bar{y}_{6} \leq 3
$$

defines a facet of $K$. (In fact it is a so-called "general LCI", see [7, 10]). So the inequality $y_{2}+y_{3}+y_{4}+2 y_{5}+2 y_{6} \geq 4$ is valid for $P^{=}$. One can check that $\phi(0)=0, \phi(1)=u_{2}=3, \phi(2)=u_{2}+u_{3}=6, \phi(3)=u_{2}+u_{3}+u_{4}=9$ and $\phi(4)=d=11$. This yields $\delta=(11-9) /(4-3)=2$. Therefore, the RKI

$$
x_{2}+x_{3}+x_{4}+x_{5}+x_{6} \leq 3+2\left(y_{2}+y_{3}+y_{4}+2 y_{5}+2 y_{6}\right)
$$

is valid for $P$. Moreover, the graph $G$ contains the edge $\{i, j\}$ for $i=2,3,4$ and $j=5,6$, since $3+5=8<9$. So $G$ is connected, and the RKI defines a facet of $P$.

\subsection{Special cases}

We now consider the particular RKIs that are obtained when the given valid inequality for $K$ is a cover or extended cover inequality. It turns out that, in both of these special cases, there is a closed-form expression for $\delta$.

Let $F \subseteq N$ be a flow cover, and let $K$ be defined as in (6). A set $C \subseteq F$ is a cover for $K$ if and only if

$$
\sum_{j \in C} u_{j}>\sum_{j \in F} u_{j}-d
$$

Moreover, a cover $C$ is minimal if and only if

$$
\sum_{j \in C \backslash\{k\}} u_{j} \leq \sum_{j \in F} u_{j}-d \quad(\forall k \in C) .
$$


Given a minimal cover $C$, the cover inequality $\sum_{j \in C} \bar{y}_{j} \leq|C|-1$ is valid and supporting for $K$. Complementing, we find that the inequality $\sum_{j \in C} y_{j} \geq 1$ is valid and supporting for $P^{=}$.

Now, if $\sum_{j \in C} y_{j}=0$, the largest value that $\sum_{j \in F} u_{j} y_{j}$ can take is $\sum_{j \in F \backslash C} u_{j}$. Moreover, from (8), this quantity is less than $d$. So $\phi(0)=$ $\sum_{j \in F \backslash C} u_{j}<d$. On the other hand, when $\sum_{j \in C} y_{j}=1$, the largest value that $\sum_{j \in F} u_{j} y_{j}$ can take is $u^{*}+\sum_{j \in F \backslash C} u_{j}$, where $u^{*}=\max _{j \in C} u_{j}$. From (9), this quantity is larger than $d$. So $\phi(t)=d$ for $t=1, \ldots,|C|$. This implies that $\delta=d-\sum_{j \in F \backslash C} u_{j}$, and the resulting RKI is

$$
\sum_{j \in F} x_{j} \leq d+\left(d-\sum_{j \in F \backslash C} u_{j}\right)\left(\sum_{j \in C} y_{j}-1\right) .
$$

As for extended cover inequalities, we set $E=\left\{j \in F \backslash C: u_{j} \geq u^{*}\right\}$, and the extended cover inequality takes the form

$$
\sum_{j \in C \cup E} \bar{y}_{j} \leq|C|-1 .
$$

Complementing yields the following valid inequality for $K$ :

$$
\sum_{j \in C \cup E} y_{j} \geq 1+|E|
$$

Using the same argument as before, we have $\phi(0)=\sum_{j \in F \backslash(C \cup E)} u_{j}$ and $\phi(t)=d$ for $t \geq|E|+1$. Now, let $S(t)$ denote the sum of the $t$ largest $u_{j}$ values for $j \in E$. One can check that $\phi(t)$ equals $\phi(0)+S(t)$ for $t=0, \ldots,|E|$. In particular, $\phi(|E|)=\sum_{j \in F \backslash C} u_{j}<d$. Now, observe that $\phi(t)-\phi(t-1) \geq u^{*}$ for $t=1, \ldots,|E|$, but

$$
\phi(|E|+1)-\phi(|E|)=d-\sum_{j \in F \backslash C} u_{j} \leq u^{*},
$$

where the inequality on the right is implied by (9). So $\delta=d-\sum_{j \in F \backslash C} u_{j}$, and the resulting RKI is :

$$
\sum_{j \in F} x_{j} \leq d+\left(d-\sum_{j \in F \backslash C} u_{j}\right)\left(\sum_{j \in C \cup E} y_{j}-1-|E|\right) .
$$

One can check that the RKI (11) dominates the RKI (10).

\section{Single-Node Flow Polytope}

We now extend our results to the single-node flow polytope, which we will again denote by $P$. This case turns out to be considerably more complicated. 
Let $U^{+}$and $L^{+}$be disjoint subsets of $N^{+}$, and let $U^{-}$and $L^{-}$be disjoint subsets of $N^{-}$. The sets $U^{-}, L^{+}$and $L^{-}$are permitted to be empty, but $U^{+}$must be non-empty.

Let $P \geq$ be the convex hull of the feasible solutions that satisfy the inequality

$$
\sum_{j \in U^{+}} x_{j}-\sum_{j \in L^{-}} x_{j} \geq d+\sum_{j \in U^{-}} u_{j}-\sum_{j \in L^{+}} \ell_{j}
$$

Since $P^{\geq}$is contained in $P$, every $(x, y) \in P^{\geq}$must satisfy the trivially valid inequality

$$
\sum_{j \in U^{+} \cup L^{+}} x_{j} \leq \sum_{j \in N^{-}} x_{j}+d .
$$

Multiplying (12) by 2 and adding (13) we get

$$
\sum_{j \in U^{+} \cup N^{-}} x_{j}-\sum_{j \in L^{+} \cup L^{-}} x_{j}-\sum_{j \in L^{-}} x_{j} \geq d+2 \sum_{j \in U^{-}} u_{j}-2 \sum_{j \in L^{+}} \ell_{j} .
$$

Hence, all points in $P^{\geq}$satisfy the inequality

$$
\sum_{j \in U^{+} \cup\left(N^{-} \backslash L^{-}\right)} x_{j}-\sum_{j \in L^{+} \cup L^{-}} x_{j} \geq d+2 \sum_{j \in U^{-}} u_{j}-2 \sum_{j \in L^{+}} \ell_{j} .
$$

Weakening this using (4), we find that all points in $P^{\geq}$satisfy

$$
\sum_{j \in U^{+} \cup\left(N^{-} \backslash L^{-}\right)} u_{j} y_{j}-\sum_{j \in L^{+} \cup L^{-}} \ell_{j} y_{j} \geq d+2 \sum_{j \in U^{-}} u_{j}-2 \sum_{j \in L^{+}} \ell_{j} .
$$

Now, as before, let $\overline{y_{j}}$ denote $1-y_{j}$. Also let $R^{-}$denote $N^{-} \backslash\left(U^{-} \cup L^{-}\right)$. All points in $P^{\geq}$satisfy:

$$
\sum_{j \in U^{+} \cup U^{-} \cup R^{-}} u_{j} \bar{y}_{j}+\sum_{j \in L^{+} \cup L^{-}} \ell_{j} y_{j} \leq \sum_{j \in U^{+} \cup R^{-}} u_{j}-\sum_{j \in U^{-}} u_{j}+2 \sum_{j \in L^{+}} \ell_{j}-d .
$$

We now define a knapsack polytope, $K$, as the convex hull of pairs $(y, \bar{y}) \in$ $\{0,1\}^{U^{+} \cup U^{-} \cup R^{-} \cup L^{+} \cup L^{-}}$that satisfy 14 . We remark in passing that a necessary condition for $K$ to be full-dimensional is

$$
\sum_{j \in U^{+} \cup R^{-}} u_{j}+2 \sum_{j \in L^{+}} \ell_{j}>d+\sum_{j \in U^{-}} u_{j} .
$$

Next, let

$$
\sum_{j \in U^{+} \cup U^{-} \cup R^{-}} \alpha_{j} \bar{y}_{j}+\sum_{j \in L^{+} \cup L^{-}} \beta_{j} y_{j} \leq \gamma .
$$

be a supporting valid inequality for $K$ with non-negative coefficients. Complementing yields

$$
\sum_{j \in U^{+} \cup U^{-} \cup R^{-}} \alpha_{j} y_{j}-\sum_{j \in L^{+} \cup L^{-}} \beta_{j} y_{j} \geq \sum_{j \in U^{+} \cup U^{-} \cup R^{-}} \alpha_{j}-\gamma .
$$


To simplify the notation, let $\gamma^{*}=\sum_{j \in U^{+} \cup U^{-} \cup R^{-}} \alpha_{j}-\gamma$.

As in Section 3 , there exists a largest positive rational $\delta$ such that the inequality

$$
\begin{aligned}
& \sum_{j \in U^{+}} x_{j}-\sum_{j \in L^{-}} x_{j} \leq d+\sum_{j \in U^{-}} u_{j}-\sum_{j \in L^{+}} \ell_{j} \\
& \quad+\delta\left(\sum_{j \in U^{+} \cup U^{-} \cup R^{-}} \alpha_{j} y_{j}-\sum_{j \in L^{+} \cup L^{-}} \beta_{j} y_{j}-\gamma^{*}\right)
\end{aligned}
$$

is valid for $P$. This inequality is the desired RKI.

As before, in order to determine the value of $\delta$, we define an auxiliary function. For $t \in \mathbb{Z}$, let

$$
\begin{gathered}
\phi(t)=\max \left\{\sum_{j \in U^{+}} x_{j}-\sum_{j \in L^{-}} x_{j}:(3),{ }^{4},\right. \\
\left.\sum_{j \in U^{+} \cup U^{-} \cup R^{-}} \alpha_{j} y_{j}-\sum_{j \in L^{+} \cup L^{-}} \beta_{j} y_{j} \leq t, x_{j} \geq 0, y_{j} \text { binary }\right\} .
\end{gathered}
$$

Again, $\phi(t)$ is an integer-valued, non-decreasing function, but its natural domain is

$$
\mathbb{Z} \cap\left[-\sum_{L^{+} \cup L^{-}} \beta_{j}, \sum_{j \in U^{+} \cup U^{-} \cup R^{-}} \alpha_{j}\right] .
$$

One can compute the values taken by $\phi(t)$ over this domain efficiently by dynamic programming. (Details omitted for brevity.) Once the $\phi(t)$ values have been computed, one can compute the value $\delta$ as follows. Let $t^{*}$ be the minimum value of $t$ such that $\phi(t)=d+\sum_{j \in U^{-}} u_{j}-\sum_{j \in L^{+}} \ell_{j}$. (Note that $t^{*} \geq \gamma^{*}$.) Then let

$$
\delta=\min _{-\sum_{j \in L^{+} \cup L^{-}} \beta_{j} \leq t<t^{*}}\left\{\frac{d-\phi(t)}{\gamma^{*}-t}\right\} .
$$

Again, we illustrate this theory with an example.

Example 2: Let $n=7, N^{+}=\{1,2,3\}, N^{-}=\{4,5,6,7\}, d=4, u=$ $(4,3,3,2,2,2,2)$ and $\ell=(1,1,1,1,1,1,1)$. Suppose we set $U^{+}=\{2,3\}$, $L^{+}=\emptyset, U^{-}=\emptyset$ and $L^{-}=\{4\}$. The knapsack constraint is $3 y_{2}+3 y_{3}-y_{4}+$ $2 y_{5}+2 y_{6}+2 y_{7} \geq 4$. Complementing gives $3 \bar{y}_{2}+3 \bar{y}_{3}+y_{4}+2 \bar{y}_{5}+2 \bar{y}_{6}+2 \bar{y}_{7} \leq 8$. The inequality $2 \bar{y}_{2}+2 \bar{y}_{3}+y 4+\bar{y}_{5}+\bar{y}_{6}+\bar{y}_{7} \leq 5$ is valid for $K$. (Again, it is a non-simple LCI.) So the inequality $2 y_{2}+2 y_{3}-y_{4}+y_{5}+y_{6}+y_{7} \geq 2$ is valid for $P^{\geq}$. We have $\phi(-1)=-1, \phi(0)=0, \phi(1)=2, \phi(2)=3$ and $\phi(3)=4$. So $t^{*}=3$ and $\delta=1$, and we obtain the RKI $x_{2}+x_{3}-x_{4} \leq$ 
$4+\left(2 y_{2}+2 y_{3}-y_{4}+y_{5}+y_{6}+y_{7}-3\right)$. One can check that this RKI defines a facet of $P$. Other RKIs for this instance include, for example, the following:

$$
\begin{aligned}
x_{1}+x_{2}-x_{4}-x_{5} & \leq 4+\left(3 y_{1}+2 y_{2}-y_{4}-y_{5}+y_{6}+y_{7}-3\right) \\
x_{2}+x_{3}-x_{5} & \leq 4+\left(2 y_{2}+2 y_{3}+y_{4}-y_{5}+y_{6}+y_{7}-3\right) \\
x_{1}+x_{3}-x_{7} & \leq 4+\left(2 y_{1}-y_{2}+y_{3}+y_{4}+y_{5}+y_{6}-y_{7}-1\right) .
\end{aligned}
$$

One can check that these too are facet-defining.

\section{Concluding Remarks}

We have introduced new families of valid inequalities for the fixed-charge and single-node flow polytopes. The inequalities, called rotated knapsack inequalities (RKIs), are completely different to the well-known flow cover inequalites and variants. Note that our procedure can yield a huge number of RKIs, because the number of possible choices for the subsets $U^{+}, U^{-}$, etc. and the number of facets of the restricted knapsack polytope can both grow exponentially in the size of the problem. A natural topic for research is to find a necessary and sufficient condition for an RKI to be facet-defining. Another pressing question is the development of effective exact or heuristic separation algorithms for the RKIs.

\section{Acknowledgements}

The second author gratefully acknowledges financial support from the EPSRC through the STOR-i Centre for Doctoral Training under grant EP/L015692/1.

\section{References}

[1] A. Atamtürk (2001) Flow pack facets of the single node fixed-charge flow polytope. Oper. Res. Lett., 29, 107-114.

[2] A. Atamtürk (2005) Cover and pack inequalities for (mixed) integer programming. Ann. Oper. Res., 139, 21-38.

[3] E. Balas (1975) Facets of the knapsack polytope. Math. Program., 8, $146-164$.

[4] V. Chvátal (1975) On certain polytopes associated with graphs. J. Combin. Th. B, 18, 138-154.

[5] M. Conforti, G. Cornuéjols \& G. Zambelli (2010) Polyhedral approaches to mixed integer linear programming. In M. Juenger et al. (eds.) 50 Years of Integer Programming, pp. 343-385. Heidelberg: Springer. 
[6] W. Cook (2010) Fifty-plus years of combinatorial integer programming. In M. Juenger et al. (eds.) 50 Years of Integer Programming, pp. 387430. Heidelberg: Springer.

[7] Z. Gu, G.L. Nemhauser \& M.W.P. Savelsbergh (1998) Lifted cover inequalities for 0-1 integer programs: computation. INFORMS J. Comput., 10, 427-437.

[8] Z. Gu, G.L. Nemhauser \& M.W.P. Savelsbergh (1999) Lifted flow cover inequalities for mixed 0-1 integer programs. Math. Program., 85, 439467.

[9] Z. Gu, G.L. Nemhauser \& M.W.P. Savelsbergh (1999) Sequenceindependent lifting in mixed integer programming. J. Comb. Optim., $4,109-129$.

[10] K. Kaparis \& A.N. Letchford (2010) Separation algorithms for 0-1 knapsack polytopes. Math. Program., 124, 69-91.

[11] M.W. Padberg, T.J. Van Roy \& L.A. Wolsey (1985) Valid linear inequalities for fixed charge problems. Oper. Res., 33, 842-861.

[12] J.I.A. Stallaert (1997) The complementary class of generalized flow cover inequalities. Disc. Appl. Math., 77, 73-80.

[13] T.J. Van Roy \& L.A. Wolsey (1986) Valid inequalities for mixed 0-1 programs. Disc. Appl. Math., 14, 199-213.

[14] T.J. Van Roy \& L.A. Wolsey (1987) Solving mixed integer programming problems using automatic reformulation. Oper. Res., 35, 45-57.

[15] R. Weismantel (1997) On the 0-1 knapsack polytope. Math. Program., $77,49-68$.

[16] L.A. Wolsey (1975) Faces for a linear inequality in 0-1 variables. Math. Program., 8, 165-178. 